\title{
CREATION OF REFERENCE SPECIMENS OF THE SORPTION PROPERTIES OF NANOPOROUS SILICA GELS
}

E. P. Sobina, ${ }^{1}$ I. S. Puzyrev, ${ }^{2}$

UDC 543.08

\author{
S. V. Medvedevskikh, ${ }^{1}$ M. Yu. Medvedevskikh, ${ }^{1}$ \\ M. P. Krasheninina, ${ }^{1}$ L. V. Adamova, ${ }^{3}$ \\ L. K. Neudachina, ${ }^{3}$ and Yu. G. Yatluk ${ }^{2}$
}

A technique for the synthesis of nanoporous silica gels is developed. Reference specimens are created and an estimate of their metrological characteristics is performed.

Keywords: nanoporous silica gels, reference specimens.

The development of nanotechnologies and their widespread introduction into science, technology, industry, and quality control of products must be supported with improved methods and means of measurement. The use of reference specimens is one of the more important means of metrological assurance. There are now many enterprises in Russia involved in the production of nanoporous substances and materials (sorbents, catalysts, zeolites, etc.). Reference specimens of sorption properties with the following certified characteristics are needed: quality control at all stages of the production process: specific surface area, specific volume of pores, and mean and most common diameters of the pores.

The present study is devoted to the creation of reference specimens of the sorption properties of nanoporous silica gels performed by organizations that are members of the regional division of the Center for Metrological Assurance and Evaluation of the Conformity of Nanotechnologies and Products of the Nanotechnology Industry in the Ural Federal district.

We will use the template colloidal solution - gel method. Organic bases, such as polyethylenimine (PEA) produced by the firm of Fluka with average molecular weight $50 \cdot 10^{3}$ amu as well as a mixture of primary distilled amines not previously investigated by this method were selected as the templates. The mean composition determined potentiometrically corresponds to $\mathrm{C}_{12} \mathrm{H}_{25} \mathrm{NH}_{2}$; from the results of gas-liquid chromatography, the mixture contains $80 \%$ dodecylamine. The mixture of primary distilled amines is used without additional purification.

Synthesis of silica gels (fabrication of reference specimens). The synthesis procedure consists of the following steps. Amine is dissolved in ethyl alcohol or a water-alcohol solution, and tetraethylorthosilicate (from the firm of Fluka, mass fraction of basic substance 98\%) then added in the mixing process to form a gel. The resulting gel is mixed in Petri dishes and dried in a drying oven for $24 \mathrm{~h}$ at $70^{\circ} \mathrm{C}$ until the mass of the specimen has become invariant. The specimen is then placed in flat-bottom quartz bowls and calcined in open air. The synthesis conditions are summarized in Table 1.

Investigation of structure of reference specimens. The porosity characteristics and sorption properties (specific surface area, specific volume of pores, dimension of pores, distribution of pores by dimension) of specimens of silica gels of interme-

\footnotetext{
${ }^{1}$ Ural Research Institute of Metrology, Yekaterinburg, Russia; e-mail: sobina_egor@uniim.ru.

${ }^{2}$ Institute for Organic Synthesis, Ural Branch, Russian Academy of Sciences; e-mail: igor.puzyrev@mail.ru.

${ }^{3}$ Institute of Natural Sciences, Yeltsin Ural Federal University, Yekaterinburg, Russia.
}

Translated from Izmeritel'naya Tekhnika, No. 6, pp. 25-27, June, 2013. Original article submitted February 4, 2013. 
TABLE 1. Synthesis Conditions of Reference Specimens of Sorption Properties of Nanoporous Silica Gels

\begin{tabular}{|c|c|c|c|c|c|}
\hline \multirow{2}{*}{$\begin{array}{c}\text { Index of reference } \\
\text { specimen }\end{array}$} & Amine & \multicolumn{2}{|c|}{ Hold time at temperature, min } & \multicolumn{2}{|c|}{ Volume of reagent, $\mathrm{cm}^{3}$} \\
\cline { 2 - 6 } & PEA & $500^{\circ} \mathrm{C}$ & $70{ }^{\circ} \mathrm{C}$ & $\mathrm{C}_{2} \mathrm{H}_{5} \mathrm{OH}$ & 236.0 \\
\hline NMS-1 & 180 & 5 & 257.3 & 257.3 \\
\hline NMS-2 & $\mathrm{C}_{12} \mathrm{H}_{25} \mathrm{NH}_{2}$ & - & 50 & 257.3 & - \\
\hline NMS-3 & $\mathrm{C}_{12} \mathrm{H}_{25} \mathrm{NH}_{2}$ & 180 & 5 & 51.5 \\
\hline NMS-4 & $\mathrm{C}_{12} \mathrm{H}_{25} \mathrm{NH}_{2}$ & 180 & 5 & \\
\hline
\end{tabular}

TABLE 2. Metrological Characteristics of Reference Specimens (certification with respect to nitrogen at $T=-196^{\circ} \mathrm{C}$ by gasadsorption method)

\begin{tabular}{|c|c|c|c|c|c|c|c|c|}
\hline \multirow{2}{*}{$\begin{array}{c}\text { Certified } \\
\text { characteristic }\end{array}$} & \multicolumn{2}{|c|}{ NMS-1 } & \multicolumn{2}{|c|}{ NMS-2 } & \multicolumn{2}{|c|}{ NMS-3 } & \multicolumn{2}{|c|}{ NMS-4 } \\
\hline & $A$ & $\delta A, \%$ & $A$ & $\delta A, \%$ & $A$ & $\delta A, \%$ & $A$ & $\delta A, \%$ \\
\hline$S_{\mathrm{s}}, \mathrm{m}^{2} / \mathrm{g}$ & 438.900 & \pm 1.4 & 886.500 & \pm 1.7 & 1055.000 & \pm 2.0 & 1100.800 & \pm 1.6 \\
\hline$V_{\mathrm{s}}, \mathrm{cm}^{3} / \mathrm{g}$ & 0.354 & \pm 3.0 & 0.814 & \pm 3.2 & 0.823 & \pm 2.9 & 1.043 & \pm 3.0 \\
\hline$d_{\mathrm{av}}, \mathrm{nm}$ & 3.270 & \pm 3.9 & 3.680 & \pm 2.6 & 3.110 & \pm 2.8 & 3.800 & \pm 2.4 \\
\hline $\begin{array}{l}D \text { (from adsorption branch of } \\
\text { isotherm), } \mathrm{nm}\end{array}$ & 1.850 & \pm 2.0 & 1.740 & \pm 1.8 & 2.210 & \pm 2.1 & 1.860 & \pm 2.3 \\
\hline $\begin{array}{l}D \text { (from desorption branch of } \\
\text { isotherm), nm }\end{array}$ & 3.690 & \pm 2.4 & 3.460 & \pm 1.8 & 3.710 & \pm 2.0 & 3.440 & \pm 1.8 \\
\hline$A_{w}\left(p / p_{0}=0.05\right), \mathrm{cm}^{3} / \mathrm{g}$ & 127.100 & \pm 3.9 & 185.600 & \pm 2.5 & 165.000 & \pm 2.8 & 207.000 & \pm 2.6 \\
\hline$A_{w}\left(p / p_{0}=0.40\right), \mathrm{cm}^{3} / \mathrm{g}$ & 157.800 & \pm 4.0 & 312.600 & \pm 1.8 & 390.600 & \pm 2.1 & 379.000 & \pm 2.4 \\
\hline$A_{w}\left(p / p_{0}=0.99\right), \mathrm{cm}^{3} / \mathrm{g}$ & 415.900 & \pm 3.2 & 784.600 & \pm 1.9 & 539.400 & \pm 2.5 & 1099.000 & \pm 2.9 \\
\hline
\end{tabular}

diate porosity were measured by a gas-adsorption method with the use of a TriStar 3020 analyzer [1]. The microstructure of these specimens was studied by means of a CrossBeam System scanning electron microscope functioning as a component of an Auriga workstation. The results indicate that these specimens constitute agglomerates of spherical particles measuring 60-800 nm with pores $2-10 \mathrm{~nm}$ (Table 2). Here and below, the following notation has been introduced: $A_{w}$, sorption capacity in the case of relative pressure $p / p_{0}$ reduced to normal conditions $\left(T_{0}=273.15 \mathrm{~K}, p_{0}=101.325 \mathrm{kPa}\right.$, $p$, current pressure of nitrogen); $A$, certified value of characteristic of reference specimen; $\delta A$, relative error in $A$ with 0.95 confidence level; $S_{\mathrm{s}}$, specific surface area; $V_{\mathrm{s}}$, specific volume of pores; and $d_{\mathrm{av}}$ and $D$, mean and most common diameters of pores, respectively.

Determination of metrological characteristics of reference specimens. A measurement technique was developed in order to determine the sorption properties of nanoporous silica gels [2]. Through the use of the technique used in measurements of the sorption properties by means of a gas-adsorption method it is possible to obtain results in ranges of measurement and with characteristics of the relative error that do not exceed the values specified in Table 3, where $\sigma_{r}$ is the relative standard deviation of recurrence; and $\delta_{\text {sys }}$ and $\delta$, relative nonexcluded systematic and relative total error with $P=0.95$, respectively. The traceability of the measurement results is established by means of the following approved types entered into the register of reference specimens:

State Reference Specimen GSO 9445-2009 (ERM-FD 107/BAM-P107): interval of certified value of $S_{\mathrm{s}}, 600-620 \mathrm{~m}^{2} / \mathrm{g}$; absolute error $\pm 14.5 \mathrm{~m}^{2} / \mathrm{g}$; interval of certified value of $V_{\mathrm{s}}, 0.200-0.300 \mathrm{~cm}^{3} / \mathrm{g}$, absolute error $\pm 0.005 \mathrm{~cm} / \mathrm{g}$; interval of certified value of $d_{\text {av }} 0.80-0.90 \mathrm{~nm}$, absolute error $\pm 0.05 \mathrm{~nm}$. 
TABLE 3. Metrological Characteristics of Technique for Measurement of Sorption Properties by Gas-Adsorption Method

\begin{tabular}{|c|c|c|c|c|}
\hline Measured quantity & Range of measurement & $\sigma_{r}, \%$ & $\delta_{\text {sys }}, \%$ & $\delta, \%$ \\
\hline$A_{w}, \mathrm{~cm}^{3} / \mathrm{g}$ & $10-2000$ & 1.1 & 2.3 & 3.4 \\
\hline$S_{\mathrm{s}}, \mathrm{m}^{2} / \mathrm{g}$ & $0.1-2000.0$ & 1.4 & 1.0 & 3.0 \\
\hline$V_{\mathrm{s}}, \mathrm{cm}^{3} / \mathrm{g}$ & $0.02-2.00$ & 2.7 & 2.6 & 6.1 \\
\hline$d_{\mathrm{av}}, \mathrm{nm}$ & $0.4-100.0$ & 2.8 & 2.6 & 6.3 \\
\hline$D, \mathrm{~nm}$ & $2-100$ & 2.5 & 1.8 & 5.4 \\
\hline
\end{tabular}

GSO 9446-2009 (CRM BAM PM-104): interval of certified value of $S_{\mathrm{s}}, 70-80 \mathrm{~m}^{2} / \mathrm{g}$, absolute error $\pm 1.0 \mathrm{~m}^{2} / \mathrm{g}$; interval of certified value of $V_{\mathrm{s}}, 0.150-0.300 \mathrm{~cm}^{3} / \mathrm{g}$, absolute error $\pm 0.014 \mathrm{~cm}^{3} / \mathrm{g}$; interval of certified value of hydraulic radius of pores, $5.00-6.00 \mathrm{~nm}$, absolute error $\pm 0.21 \mathrm{~nm}$.

Investigation of homogeneity of material of reference specimens performed according to [3-6]. $N=6$ samples of material of the reference specimens were randomly selected and $j=3$ measurements of each certified characteristic were performed in accordance with a certified technique. The measured values of each such characteristic were estimated on the basis of $[6,7]$.

An investigation of the stability of reference specimens, expressed in the invariance of a certified characteristic over time with storage and application conditions observed, was conducted by an isochronous method (artificial (accelerated) ageing) in accordance with the recommendations of $[7,8]$. The reference specimens were held at an elevated temperature of $115^{\circ} \mathrm{C}$ and the values of the characteristics were then measured and compared to the initial values. Processing of the results demonstrated that they do not differ significantly before and after ageing.

The algorithms proposed in $[7,8]$ were used to determine the limits of the absolute errors of the certified values of the reference specimens as well as the expanded uncertainty. Data for approval of the type of these reference specimens were prepared from the results of this research in accordance with the requirements set forth in $[9,10]$. Values and limits of their absolute errors for the first batch of reference specimens are presented in Table 2 (two batches have now been produced).

In order to evaluate the suitability of a reference specimen and investigate its commutativity, measurements were performed at laboratories, such as the laboratory at the Aspekt Association (Moscow) by means of an ASAP 2020 analyzer; metrological assurance of the nanotechnology industry, spectral methods of analysis and reference specimens performed at the Ural Research Institute of Metrology (UNIIM) with the use of an ASAP 2020 MR analyzer, and sorption performed at the Ural Federal University with the use of an TriStar 3020 analyzer.

The results of measurements of the porosity characteristics of the sorption properties obtained in different laboratories and on different equipment are in satisfactory agreement with the certified values of the sorption characteristics of newly developed reference specimens within the range of certified values of the measurement error. Investigations demonstrated the suitability of the newly developed reference specimen for testing and calibration of different types of gas-adsorption analyzers.

State Calibration Standard GSO 9735-2011 of the sorption properties of nanoporous silica gels (NMS set of reference specimens maintained at the UNIIM), which does not have any analogs and is included in the State Register of Approved Types of Reference Specimens of the Russian Federation, was created from the results of the investigations. This reference specimen is a nanoporous disperse powder of silica gels that is stored in 5-g packages in airtight jars. The shelf life is 5 years. The newly developed reference specimen is designed for calibration of measuring instruments and to assure the traceability of measurements of the sorption characteristics by the gasoadsorption method and control of the error of the measurement techniques as well as for testing and other types of metrological control where the metrological characteristics of the reference specimen correspond to the requirements of the control procedures. Data on reference specimens are included in the Federal Information Bank for Assurance of the Uniformity of Measurements.

GSO 9735-2011 is recognized as an inter-governmental reference specimen by decision of the Inter-governmental Council on Standardization, Metrology, and Certification of May 24, 2012, Protocol No.41-2012, and was added to the reg- 
ister of Inter-governmental Reference Specimens as No. 1783:2012; it is accepted for use without restrictions in Azerbaijan, Armenia, Belarus, Kazakhstan, Kirghizia, Tajikistan, Turkmenistan, Uzbekistan, and Ukraine.

Studies on the production of new batches and new types of reference specimens of sorption properties are now continuing at metrological assurance laboratories of the nanotechnology industry as well as in studies of spectral methods of analysis and reference specimens at the Ural Research Institute of Metrology.

The present study was conducted within the framework of State Contract 120-150, Development of Procedures Component of the Regional Division of the Center of Metrological Assurance and Evaluation of Correspondence of Nanotechnologies and Output of the Nanotechnology Industry in the Ural Federal District. The study was completed with the support of Program No. 12-P-234-2003 of the Presidium of the Russian Academy of Sciences.

\section{REFERENCES}

1. S. Gregg and C. Singh, Adsorption, Specific Surface, Porosity [Russian translation], Mir, Moscow (1984).

2. FR.1.27.2011.10971, GSI. Technique of Measurements of Specific Surface Area, Specific Volume of Pores, and Dimension of Pores by Method of Physical Sorption of Nanoporous Modified Silica Gels.

3. V. V. Kazantsev et al., "Development of techniques for estimating nonuniformity in the creation of reference specimens of chemical compounds and properties of nanomaterials," Stand. Obraztsy, No. 1, 29-43 (2011).

4. V. V. Kazantsev et al., "Development of techniques for estimating nonuniformity in the creation of reference specimens of chemical compounds and properties of nanomaterials," Stand. Obraztsy, No. 2, 37-48 (2011).

5. V. V. Kazantsev et al., "Development of techniques for estimating nonuniformity in the creation of reference specimens of chemical compounds and properties of nanomaterials," Stand. Obraztsy, No. 3, 61-70 (2011).

6. RMG 53-2002, GSI. Reference Specimens. Estimation of Metrological Characteristics with the Use of References and Reference Measuring Instruments.

7. RMG 93-2009, GSI. Estimation of Metrological Characteristics of Reference Specimens.

8. R 50.2.058-2007, GSI. Estimation of Uncertainty of Certified Values of Reference Specimens.

9. MI 3300-2010, GSI. Recommendations for Preparation, Presentation, and Review of Results of Tests of Reference Specimens for Purposes of Approval of Type.

10. GOST 8.315-97, GSI. Reference Specimens of a Composition and Properties of Substances and Materials. Basic Assumptions. 